Sharif University of Technology
Scientia Iranica
Transactions E: Industrial Engineering
hCIENTIA

\title{
A novel risk assessment approach using Monte Carlo simulation based on co-occurrence of risk factors: A case study of a petrochemical plant construction
}

\author{
B. Ostadi* and S. Abbasi Harofteh \\ Faculty of Industrial and Systems Engineering, Tarbiat Modares University, Tehran, Iran.
}

Received 25 February 2020; received in revised form 4 June 2020; accepted 20 July 2020

\author{
KEYWORDS \\ Risk assessment; \\ Co-occurrence of risk \\ factors; \\ Risk prioritization; \\ Uncertainty; \\ Monte Carlo \\ simulation.
}

\begin{abstract}
Nowadays, due to the development of technology as well as the occurrence of unpredictable events, it is necessary to address risk management as an important part of projects and businesses. In this paper, a novel approach based on Monte Carlo simulation is proposed for risk assessment, which considers the co-occurrence of risks. In this method, the output of extended and classic Monte Carlo simulations is employed for Co-Occurrencebased Risk Assessment (CORA) and prioritization. Also, the magnitude of uncertainty in each source has been determined using the new approach. In addition to identifying and analyzing the risks, the proposed model investigates possible relationships between risks and determines the type of risk-induced impact as either resonance or reduction. Also, a system dynamic model is applied to illustrate the relationships among the risks. Finally, this method is utilized for a petrochemical project. Five risks including temperature, rain, labor, cost, and inflation are considered in this project. Based on the numerical results, the most important risk is inflation. Also, there is a significant difference between the result of the proposed model and the model outcome, which had previously ignored the co-occurrence of risks. CORA helps managers consider all aspects of risks and make a better decision.
\end{abstract}

(C) 2022 Sharif University of Technology. All rights reserved.

\section{Introduction}

Nowadays, it is important to address risk management due to the rapid development of science and technology [1] and consequently, unpredictable and complicated conditions of the industrialized world. In this condition, uncertainties and risky situations are on the rise and the harm of disregarding risk and its management is clear.

Risk as an uncertain condition or event may have

\footnotetext{
*. Corresponding author. Tel.: +982182884385;

Fax: +982182884385

E-mail address: bostadi@modares.ac.ir (B. Ostadi)
}

positive or negative impacts on the final objective of a project [2].

In another definition, risk is defined as the result of uncertainty in the system which may cause either financial profit or economic loss [3].

Risk management is a systematic process with six steps consisting of planning, risk identification, qualitative risk analysis, quantitative risk analysis, risk response planning, and risk monitoring and control. This process is indeed an determining factor in the success of a project, given that risks affect not only cost, time, and resource management but also achievement of projects' objectives. Risk impacts are of greater significance in the case of large projects such as projects with many complexities, macro-finance, long-term works, projects with many resources or unstable economic 
conditions, special environmental characteristics, and various shareholders [2].

As a result, the need for evaluating and analyzing risks is essential in all steps of completing a project. There are different techniques for risk assessment such as Monte Carlo Simulation (MCS), Extended Monte Carlo Simulation (EMCS) [4], Failure Mode and Effective Analysis (FMEA), fuzzy set, event trees, fault trees, Multi-Criteria Decision Analysis (MCDA), Markov analysis, Bayesian statistics and Bayes nets, and consequence/probability matrix [5].

Also, a number of hybrid models have been proposed that attempt to combine the benefits of the mentioned techniques including fuzzy Monte Carlo simulation [6], fuzzy ANP [7], fuzzy AHP [8], etc.

Different research studies have focused on the models of risks, risk identification and analysis, risk development models, risk assessment, and risk prioritization, which are shown in Table 1.

According to Table 1, the research scopes of risk analysis are classified into five groups. Our proposed model has three phases: risk identification, risk assessment, and risk prioritization.

There are different techniques for risk identification. For example, the risks are identified through literature review, use of Delphi technique, and interview with experts [9]. Also, the risks associated with the PPP project are identified through the use of decomposition analysis and based on the scientific, system, comparison, and operation principles [25].

Different techniques including ANP, AHP, DEMATEL, and MCS are applied to risk assessment. MCS, as a popular method, is used in different scopes of risk such as risk assessment and risk modeling. For example, Rezaie et al. introduced extended Monte Carlo simulation [4]. They proposed a rotatory algorithm in which case the classic Monte Carlo simulation comes closer to reality by considering the relationship for the project's major uncertainties.

In another research, Huang et al. extended the MCS model [11]. Their proposed model considers the uncertainties associated with the historical cost data. The result of their model proved that the achieved risk distribution significantly differed from the evaluation result of the classic MCS.

In 2017, Kim proposed fuzzy MCS and compared the results with the outcome of Monte Carlo simulation. Based on the comparison of the results of fuzzy MCS and MCS, it was found that Fuzzy Monte Carlo Simulation managed to achieve more reliable and meaningful information [6].

In the case of one of the hybrid methods, Bamakan and Dehghani Mohammadabadi [14] proposed weighted Monte Carlo simulation to perform the risk assessment of information security management system by applying MCS and AHP. Risk prioritization is important because risks are ranked based on their importance and the management performs necessary actions to deal with them.

For risk prioritization, a model based on ANP was proposed to prioritize the risks associated with mega-projects [20]. Another study used the FMEA method for prioritizing the risks associated with supply chains [21].

In the real world, the occurrence of two or more risks leads to the exacerbation or reduction of the risk effects. In this regard, no research has taken the cooccurrence of risks into account.

Therefore, traditional risk assessment methods do not consider the co-occurrence of risk factors. Cooccurrence of risks results in resonance or exacerbation of the uncertainty in each source, and the manager and decision-maker should pursue a correct strategy in this situation.

Determining logical risk values remains an important challenge that was considered in the EMCS proposed by Rezaie et al. This study focuses on the issue of risk assessment with emphasis on the cooccurrence of risks. Therefore, the outputs of EMCS and MCS are used as the inputs of the proposed method so that risk assessment can be performed.

The objectives of this study are as follows:

- A new model for risk assessment by considering the co-occurrence of risks;

- Employing extended Monte Carlo simulation for accurate simulation of possible events;

- Investigating of the impact of the occurrence of risks on each other and also, determining the type of risk impact as either resonance or reduction;

- Calculating the magnitude of uncertainty in each source using a new approach;

- Designing a dynamic system model for analyzing the relationships between risks and cause-and-effect loops.

In the next section, MCS is explained. The proposed model, based on the co-occurrence of risks, is presented in Section 3. The numerical results and the proposed system dynamic model are presented in Sections 4 and 5. Finally, the last section covers the conclusion and further research suggestions.

\section{Monte Carlo simulation method}

Monte Carlo simulation, as a powerful technique in risk assessment, considers both threats and opportunities simultaneously. It also investigates the probability of the selection of different criteria. Monte Carlo simulation is used by risk assessors as a statistical technique to assess uncertainty $[4,26]$. 
Table 1. Classification of the papers based on risk methodology.

\begin{tabular}{c} 
Research \\
area in risk \\
management \\
\hline \\
Risk \\
identification \\
and analysis
\end{tabular}

Fuzzy ANP, fuzzy DEMATEL, and fuzzy TOPSIS methods

$$
\text { Fuzzy ANP }
$$

FMECA, ANP, AHP

Monte Carlo simulation Development
of model

Monte Carlo simulation

Fuzzy analytic network process

Monte Carlo
simulation

DEMATEL, ANP
It is focused on the identification and prioritization of risk of a construction project. Financial risks and project management risks were identified as the most important risks among eleven groups of risks categorized by an expert's opinion. This paper proposed some strategies to reduce these risks. The important risks were assessed by the fuzzy ANP, fuzzy DEMATEL, and fuzzy TOPSIS methods. The proposed model is appropriate for risk prioritization related to cost, time, and quality.

One hundred sixteen main risks were identified and fuzzy ANP, time, quality, safety, cost, and environmental effects were ranked as five main evaluation indices in the project of big 18-inch pipeline repair. The finding of the study illustrated that sanction, inflation, rapid changes in the rate of materials, equipment and exchange rate, and the impossibility of having access to transaction for supplying foreign goods were identified as critical risks.

A new approach to improving safety based on failure mode effect and criticality analysis technique (FMECA) was proposed. The two essential purposes of this study include (1) a model based on the effectiveness of FMECA and (2) a method to select the best mix of failures to be repaired regarding the budget considered by the firm.

In this paper, the interdependency of the uncertainties was considered in extended Monte Carlo simulation and thus, the Monte Carlo simulation came closer to reality. It improved classic Monte Carlo simulation.

This study proposed an extended Monte Carlo simulation that considered the uncertainty of history cost to analyze aircraft development cost risk. Also, its process of simulation covers the phase of the building model. The results of comparing the proposed method with classic Monte Carlo simulation show significant differences.

This study proposed a fuzzy Monte Carlo simulation and compared it with Monte Carlo. The comparison showed that

[6] the proposed model considered the stochastic and epistemic uncertainty and presented a family of probability distributions and more reliable information for decision-makers.

This study assessed the risks of implementing Enterprise

[12] Resource Planning (ERP) based on fuzzy analytic network process. Lack of management support and assistance was identified as a critical risk for a successful implementation of ERP.

A model was presented based on a DEMATEL and ANP for risk assessment in oil and gas construction projects. Since the interrelationships of factors in traditional models of risk assessment were not considered, their proposed model was [13] capable of creating a structural relationship between various effective factors to visualize complex correlations.

The results indicated that the most important dimensions based on high interrelationships with other dimensions were financial and technical dimensions. Environmental risk factors were identified as important factors that affected other factors. 
Table 1. Classification of the papers based on risk methodology (continued).

\begin{tabular}{|c|c|c|c|}
\hline $\begin{array}{c}\text { Research } \\
\text { area in risk } \\
\text { management }\end{array}$ & A focused model & Refs. & Main results and findings \\
\hline \multirow{6}{*}{$\begin{array}{c}\text { Risk } \\
\text { assessment }\end{array}$} & $\begin{array}{l}\text { Monte Carlo } \\
\text { simulation \&AHP }\end{array}$ & [14] & $\begin{array}{l}\text { This study proposed a stochastic risk assessment model of } \\
\text { information security based on AHP and Monte Carlo simula- } \\
\text { tion. The proposed model became closer to reality in the risk } \\
\text { analysis process because of defining probability distribution } \\
\text { for the level of vulnerabilities and the probability of their } \\
\text { occurrence }\end{array}$ \\
\hline & Fuzzy-AHP & {$[15]$} & $\begin{array}{l}\text { A two-stage fuzzy-AHP model was proposed to develop a risk } \\
\text { assessment model for implementing green initiatives in the } \\
\text { fashion supply chain }\end{array}$ \\
\hline & $\begin{array}{l}\text { Monte Carlo } \\
\text { simulation }\end{array}$ & {$[16]$} & A Monte Carlo simulation was used to assess risks. \\
\hline & $\begin{array}{l}\text { Monte Carlo } \\
\text { simulation }\end{array}$ & {$[17]$} & $\begin{array}{l}\text { A Monte Carlo simulation was presented based dual-interval } \\
\text { stochastic programming. }\end{array}$ \\
\hline & $\begin{array}{c}\text { Fuzzy group } \\
\text { decision making }\end{array}$ & {$[18]$} & $\begin{array}{l}\text { The bridge risk was identified by developing a Fuzzy Group } \\
\text { Decision Making (FGDM). }\end{array}$ \\
\hline & $\begin{array}{l}\text { Monte Carlo } \\
\text { simulation }\end{array}$ & [19] & $\begin{array}{l}\text { The risks of Iranian bridge construction industry were identi- } \\
\text { fied and assessed by Monte Carlo simulation. The most crucial } \\
\text { risk factor that negatively influenced projects was financial } \\
\text { risk. }\end{array}$ \\
\hline \multirow{3}{*}{$\begin{array}{l}\text { Risk } \\
\text { prioritization }\end{array}$} & ANP & {$[20]$} & $\begin{array}{l}\text { A new risk prioritization of transportation megaprojects was } \\
\text { done at the construction stage with ANP and a new Risk } \\
\text { Priority Index. Technical, economic, political, environmental, } \\
\text { and social risks were the most important risks, in order. }\end{array}$ \\
\hline & $\begin{array}{l}\text { FMEA } \\
\text { Multi-stage Fuzzy }\end{array}$ & {$[21]$} & Risk prioritization of supply chain based on FMEA was done. \\
\hline & $\begin{array}{l}\text { Cognitive Map } \\
\text { (FCM) method, } \\
\text { Process Failure } \\
\text { Mode and Effects } \\
\text { Analysis (PFMEA) }\end{array}$ & {$[22]$} & $\begin{array}{l}\text { FCM and PFMEA were employed for identification and } \\
\text { prioritization of failure of the production process of the food } \\
\text { industry. }\end{array}$ \\
\hline \multirow{2}{*}{$\begin{array}{l}\text { Risk } \\
\text { modeling }\end{array}$} & $\begin{array}{l}\text { Bow-Tie analysis, } \\
\text { optimization techniques }\end{array}$ & [23] & $\begin{array}{l}\text { A model was proposed based on Bow-Tie analysis and opti- } \\
\text { mization for modeling and mitigating the risk of the supply } \\
\text { chain. }\end{array}$ \\
\hline & $\begin{array}{l}\text { Interpretive } \\
\text { ranking process, } \\
\text { system dynamics }\end{array}$ & {$[24]$} & $\begin{array}{l}\text { Interpretive Ranking Process and System Dynamics were } \\
\text { employed to model risk factors of an Indian construction } \\
\text { project. Based on the result, the risk factor dimension } \\
\text { 'construction management' had a high probability to arise over } \\
\text { the construction phase. }\end{array}$ \\
\hline
\end{tabular}

Monte Carlo simulation has become too appealing and available for different applications. Also, the technique has become a popular quantitative method that produces probability distributions for risks [26].

In this paper, the output of the Monte Carlo simulation is used as the input of the proposed model.

Monte Carlo simulation consists of 5 steps as follows [4]:
Step 1: Determine the distribution function of uncertainties based on the available data and experts' opinions;

Step 2: Segment the area below the curve into equal squares based on the number of simulation runs;

Step 3: Initialize each uncertainty by a random number at the interval of $[1,100]$; 


$$
\begin{cases}(\max (1,(A-5 n)), \min (1,(A+5 n)) & \text { Straight impact } \\ (\max (1,100-(A+5 n))), \min (100,100-(A-5 n)) & \text { Inverse impact } \\ {[1,100]} & \text { No dependency }\end{cases}
$$

Step 4: Calculate the value of each uncertainty similar to the referenced study [4] (for more details, refer to [4]);

Step 5: Go to Step 2 to move on to the next run.

While MCS enjoys many benefits and applications as a risk assessment, it is far from reality in some cases because some uncertainties are impossible to occur in reality; in other words, the outcome should consider the action and reaction of uncertainties. Therefore, extended Monte Carlo simulation solves this problem by considering the interdependence between the uncertainties and rotatory algorithm [4].

In extended Monte Carlo simulation, instead of assigning a random value to uncertainties, they are valued with respect to the type and level of interdependencies between uncertainties.

Thus, at each stage, an uncertainty is chosen as free uncertainty, which is valued randomly; however, the values of the remaining uncertainties are determined under the control state based on the type and level of interdependencies. At each run, free uncertainty varies.

Extended Monte Carlo simulation not only eliminates inconceivable state but also analyzes the possible solution space more accurately.

The algorithm of extended Monte Carlo simulation is presented as follows [4]:

Step 1: Determine the type and level of dependency for each couple of uncertainties;

Step 2: Determine the distribution function of uncertainties according to available data and experts' opinions;

Step 3: Segment the area below the curve into equal squares based on the number of simulation runs;

Step 4: At each run, apply a rotary algorithm to choose free uncertainty;

Step 5: Initialize free uncertainty by the random number $A$ at the interval of $[1,100]$;
Step 6: Specify the control interval for all dependent uncertainties based on the type and level of dependency between free and dependent uncertainties as shown in Box I.

Step 7: Assign a random number at the controlled interval to dependent uncertainties;

Step 8: Go to Step 5 to continue with the next run.

\section{The Co-Occurrence-based Risk Assessment approach (CORA)}

Severity and probability of risks are two important factors in risk assessment. The severity of risks may increase or decrease based on the type and degree of their interdependency. Thus, the co-occurrence of risks affects the severity. To the best of our knowledge, this co-occurrence has been neglected in the classic risk assessment methods. Risks are evaluated through Eq. (1) in the classic mode:

$$
\operatorname{Risk}_{j}=S_{j} \times P_{j} \text {. }
$$

In the proposed method, risks are evaluated according to Eq. (2):

$$
\begin{aligned}
\operatorname{Risk}_{j}= & S_{j} \times P_{j} \times\left(1 \pm W_{i j}\right) \times\left(1 \pm W_{k j}\right) \\
& \times\left(1 \pm W_{m j}\right) \times\left(1 \pm W_{s j}\right) \times\left(1 \pm W_{n j}\right) \\
& \times\left(1 \pm W_{o j}\right),
\end{aligned}
$$

where $P_{j}$ is the probability of risk $j$ based on the probability distribution function of the $j$ th risk. $S_{j}$ is the severity of the $j$ th risk. The severity of risk occurrence is considered for determining $S_{j}$ using Eq. (3) (Eq. (3) is shown in Box II) which simplified the process of calculating the risk severity. $W_{i j}$ is the coefficient of the co-occurrence of the $i$ th and $j$ th risks determined at each dependency level. The steps of the proposed model are presented in Figure 1. The uncertainties are determined based on the experts' opinions in the first step given in Figure 1. Then, the distribution function

$$
S_{j}=\frac{\text { Severity of occurrence }\left(R_{j}\right) \times \text { Severity of impact }\left(R_{j}\right)}{\sum \max (\text { Severity of impact }) \times \text { Severity of occurrence }} .
$$



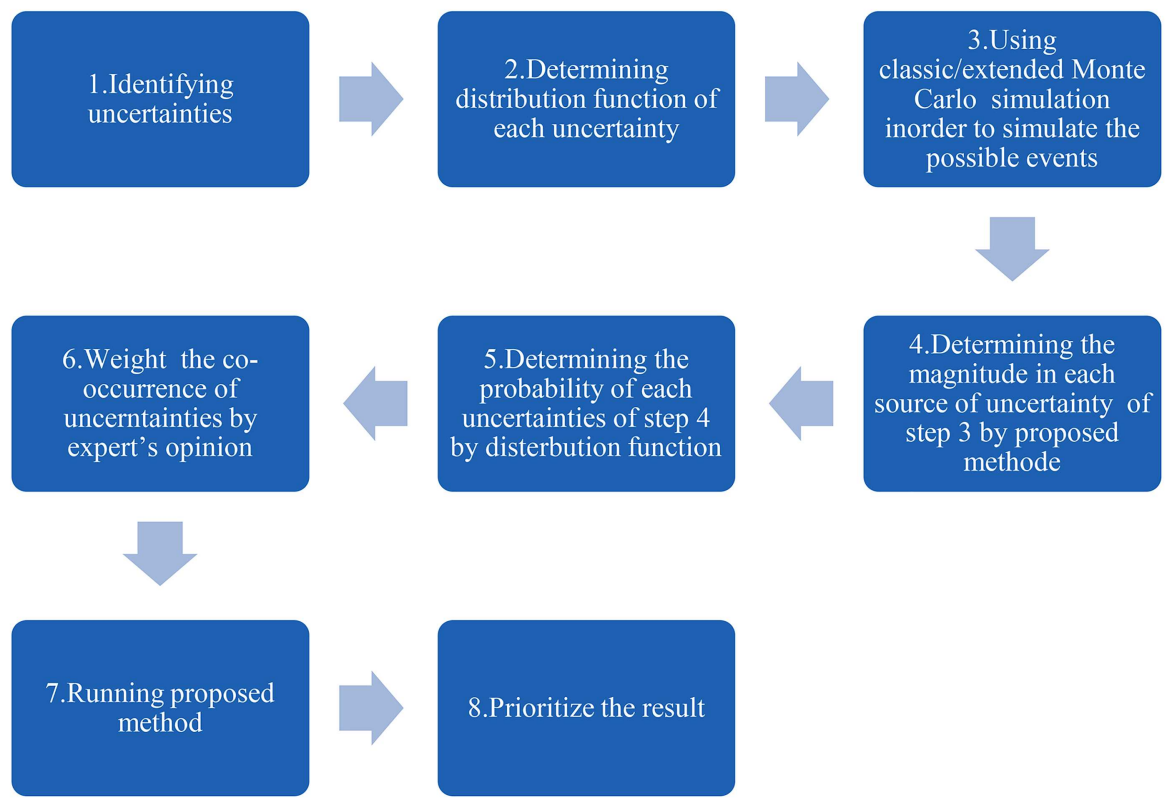

Figure 1. Steps of the CORA.

is specified for each uncertainty in an orderly fashion. In the third step, Monte Carlo or extended Monte Carlo simulation [4] is applied to produce possible values for uncertainties. Probability and severity of risks are determined in the fourth and fifth steps, respectively. In the next step, the co-occurrence of risks' coefficients is determined by experts. Finally, the proposed method is applied to risk assessment and its prioritization.

In this paper, to calculate the severity of risks, the magnitude of uncertainty in each source is measured in terms of the severity of risk occurrence and the impact severity. In previous studies, the magnitude of uncertainties is determined based on questionnaire and experts' opinions or through Monte Carlo simulation. MCS is applied because of the stochastic nature of risk assessment [13].

\section{Numerical test and results}

As mentioned earlier, risks generally occur simultaneously and the effect of each risk may vary when occurring at the same time. So, the results of risk assessment with emphasis on their co-occurrence facilitate making a more logical decision. Hence, a method functioning based on the MCS for risk assessment is proposed considering risks co-occurrence.

To evaluate the performance of the proposed model, it is customized for the project of constructing a petrochemical plant similar to the referenced study [27]. In this project, five uncertainties are considered. The uncertainties and their distribution functions are presented in Table 2 [27]. Given that this project is a petrochemically related construction
Table 2. Uncertainty distribution functions [27].

\begin{tabular}{lcc}
\hline \multicolumn{1}{c}{ Uncertainty } & $\begin{array}{c}\text { Distribution } \\
\text { function }\end{array}$ & Unit \\
\hline$x_{1}:$ Cost & $N(700,15)$ & Billion $\left(\mathrm{IRR}^{*}\right)$ \\
$x_{2}:$ Temperature & $T(10,21,31)$ & Day** \\
$x_{3}:$ Rain & $T(22,32,43)$ & Hours \\
$x_{4}:$ Labour & $N(386,15)$ & Unit \\
$x_{5}:$ Interest rate & $T(13,13.8,15.5)$ & Unit \\
\hline *: Iranian Rial; & & \\
**: Number of days when temperature is above $45^{\circ} \mathrm{C}$.
\end{tabular}

in a particular area, experts' opinions have considered uncertainties and their distributions.

To follow Step 1 of the EMCS algorithm described in Section 2, the level of dependencies between uncertainties is considered low, medium, high, and non-impact and these are quantified by 4, 2, 1, and 0 , respectively. Direct impact is shown by + . The relationships between the uncertainties are shown in Table 3 similar to the referenced studies [4,27].

The values of uncertainties are set through MCS and extended MCS. The output of MCS and extended MCS Carlo simulation based on the interval proposed by experts is categorized as the occurrence severity for each uncertainty, as shown in Table 4. The magnitude of uncertainty in each source is affected by the severity of its occurrence.

For example, imagine the inflation rate as an element of uncertainty in a project. It is obvious that the value of 10 for inflation does not have the same effect as the value of 18 . In another example, rain is an element of uncertainty in a construction project. it is clear that 35 hours of rainfall is not the same 
Table 3. Relationships between uncertainties.

\begin{tabular}{lccccc}
\hline \multicolumn{1}{c}{ Uncertainty } & Cost & Temperature & Rain & Labor & Interest rate \\
\hline$x_{1}$ : Cost & 0 & +4 & +4 & +2 & +1 \\
$x_{2}$ : Temperature & +4 & 0 & 0 & 0 & 0 \\
$x_{3}$ : Rain & +4 & 0 & 0 & 0 & 0 \\
$x_{4}$ : Labour & +2 & 0 & 0 & 0 & 0 \\
$x_{5}$ : Interest rate & +1 & 0 & 0 & 0 & 0 \\
\hline
\end{tabular}

Table 4. Categorization of MCS output based on the interval and level of impact of uncertainties.

\begin{tabular}{lcc}
\hline Uncertainty & Interval & Severity of occurrence \\
\hline \multirow{2}{*}{ Rain } & $x<25$ & Low \\
& $25-32$ & Medium \\
& $x>32$ & High \\
& $x<15$ & Low \\
Temperature & $15-20$ & Medium \\
& $x>21$ & High \\
& & \\
Interest rate & $13.6-14.2$ & Low \\
& $x>14.2$ & Medium \\
& & High \\
& $x>368$ & low \\
Labor & $350-368$ & Medium \\
& $x<350$ & High \\
& & \\
Cost & $x<718$ & Low \\
& $718-736$ & Medium \\
\hline
\end{tabular}

as 20 hours and it, thus, does not have the same impact on the end results. Therefore, determination of the uncertainty magnitude in each source affecting the severity of its occurrence leads to a more accurate definition of the risk severity.

Table 5 shows the severity of occurrence and severity of the impact for each uncertainty. Eq. (3) is applied to quantify the magnitude of uncertainty in each source.

As pointed out, the probability of the occurrence of each uncertainty is calculated by the distribution function.

Table 6 shows the coefficient of relationships between the uncertainties that occur simultaneously.

According to Table 6, the result of risk evaluation for inflation as an example is presented in Table 7 . The values of uncertainties are set through MCS and extended MCS for 1000 iterations and applied as the input of the proposed model.
Table 5. The severity and impact of occurrence.

\begin{tabular}{|c|c|c|c|}
\hline Uncertainty & $\begin{array}{c}\text { Severity } \\
\text { of occurrence }\end{array}$ & $\begin{array}{l}\text { Severity } \\
\text { of impact }\end{array}$ & $S_{j}$ \\
\hline \multirow{3}{*}{ Rain } & $\operatorname{High}(5)$ & 5 & 0.2778 \\
\hline & Medium(3) & 3 & 0.1000 \\
\hline & Low $(1)$ & 1 & 0.0111 \\
\hline \multirow{3}{*}{ Temperature } & $\operatorname{High}(5)$ & 5 & 0.2778 \\
\hline & Medium(3) & 3 & 0.1000 \\
\hline & Low $(1)$ & 1 & 0.0111 \\
\hline \multirow{3}{*}{ Interest rate } & $\operatorname{High}(5)$ & 3 & 0.1667 \\
\hline & Medium(3) & 2 & 0.0667 \\
\hline & Low $(1)$ & 1 & 0.0111 \\
\hline \multirow{3}{*}{ Labor } & $\operatorname{High}(5)$ & 8 & 0.4444 \\
\hline & Medium(3) & 6 & 0.2000 \\
\hline & $\operatorname{Low}(1)$ & 4 & 0.0444 \\
\hline \multirow{3}{*}{ Cost } & $\operatorname{High}(5)$ & 10 & 0.5556 \\
\hline & Medium(3) & 9 & 0.3000 \\
\hline & Low $(1)$ & 8 & 0.0889 \\
\hline
\end{tabular}

Moreover, the upper bound of the confidence interval at $99 \%$ is considered as the threshold of the critical value of risk and used to compare and prioritize the risks. This threshold may change in different projects based on the decision-makers' policies and strategies. Table 8 shows the results of risk analysis for all five risks.

Based on the results presented in Table 8, inflation is the most important risk that deserves greater attention. Cost, rain, temperature, and labor have the second, third, fourth, and fifth ranks, respectively.

The result of risk analysis without cooccurrence is given in Table 9 . The results show that the risks are prioritized orderly as follows: inflation, rain, temperature, cost, and labor. According to the results presented in Tables 8 and 9, there is a significant difference between the outcomes of risk analysis with and without considering the co-occurrence of risks. It is shown that the values of all risks are intensified due to their co-occurrence.

Table 10 shows the result of risk analysis consid- 
Table 6. The coefficient of co-occurrence of risks.

\begin{tabular}{lccccc}
\hline Uncertainty & Cost & Temperature & Rain & Labor & Interest rate \\
\hline Cost & 0.00 & -0.75 & 0.25 & 0.25 & 0.75 \\
Temperature & 0.75 & 0.00 & 0.25 & 0.25 & 0.00 \\
Rain & 0.25 & 0.25 & 0.00 & 0.00 & 0.00 \\
Labor & 0.25 & 0.25 & 0.00 & 0.00 & 0.00 \\
Interest rate & 0.75 & 0.00 & 0.00 & 0.00 & 0.00 \\
\hline
\end{tabular}

Table 7. Calculating the risk value of extended Monte Carlo simulation.

\begin{tabular}{ccccc}
\hline Run & $\begin{array}{c}\text { Weights of } \\
\text { occurrence }\end{array}$ & $\begin{array}{c}\text { The severity of } \\
\text { uncertainty }(\boldsymbol{S})\end{array}$ & $\begin{array}{c}\text { The probability of } \\
\text { uncertainty }(\boldsymbol{P})\end{array}$ & $\begin{array}{c}\text { Risk values } \\
\boldsymbol{w} \times \boldsymbol{S} \times \boldsymbol{P}\end{array}$ \\
\hline 1 & $((1+0.75) \times(1+0) \times(1+0) \times(1+0))$ & 0.0100 & 0.2600 & 0.0046 \\
2 & $((1+0.75) \times(1+0) \times(1+0) \times(1+0))$ & 0.0700 & 0.6200 & 0.0760 \\
3 & $((1+0.75) \times(1+0) \times(1+0) \times(1+0))$ & 0.1700 & 0.4800 & 0.1428 \\
$\vdots$ & $\vdots$ & $\vdots$ & $\vdots$ & $\vdots$ \\
1000 & $((1+0.75) \times(1+0) \times(1+0) \times(1+0))$ & 0.0100 & 0.2500 & 0.0044 \\
\hline
\end{tabular}

Table 8. Risk analysis based on extended MCS output.

\begin{tabular}{lccccc}
\hline Risk factor & Min & Mean & Max & One\% lower bound & 99\% \\
\hline Temperature & 0.0000 & 0.0078 & 0.0200 & 0.0001 & 0.0200 \\
Rain & 0.0001 & 0.0166 & 0.0333 & 0.0002 & 0.0333 \\
Inflation & 0.0009 & 0.0885 & 0.1820 & 0.0019 & 0.1806 \\
Cost & 0.0000 & 0.0104 & 0.0380 & 0.0000 & 0.0380 \\
Labor & 0.0000 & 0.0018 & 0.0062 & 0.0000 & 0.0062 \\
\hline
\end{tabular}

Table 9. Risk analysis without considering co-occurrence.

\begin{tabular}{lccccc}
\hline Risk factor & Min & Mean & Max & One\% lower bound & 99\% \\
\hline Temperature & 0.0000 & 0.0063 & 0.0160 & 0.0000 & 0.0160 \\
Rain & 0.0000 & 0.0133 & 0.0267 & 0.0002 & 0.0267 \\
Inflation & 0.0005 & 0.0505 & 0.104 & 0.0012 & 0.1032 \\
Cost & 0.0000 & 0.0025 & 0.0092 & 0.0000 & 0.0093 \\
Labor & 0.0000 & 0.0012 & 0.0041 & 0.0000 & 0.0041 \\
\hline
\end{tabular}

Table 10. The result of cooccurrence of risks with classic MCS output.

\begin{tabular}{lccccc}
\hline Risk factor & Min & Mean & Max & One\% lower bound & 99\% upper bound \\
\hline Temperature & 0.0000 & 0.0174 & 0.0199 & 0.0002 & 0.0194 \\
Rain & 0.0000 & 0.0149 & 0.0333 & 0.0002 & 0.0329 \\
Inflation & 0.0010 & 0.0848 & 0.1818 & 0.0028 & 0.1801 \\
Cost & 0.0000 & 0.0097 & 0.0380 & 0.0000 & 0.0379 \\
Labor & 0.0000 & 0.0015 & 0.0062 & 0.0000 & 0.0062 \\
\hline
\end{tabular}

ering classic MCS output and co-occurrence of risks. As demonstrated by the result, inflation is the most important risk, followed by cost, rain, temperature, and labor priorities.

The results of risk prioritization in the classic and extended MCSs by considering the co-occurrence of risks are the same, while the results of disregarding the co-occurrence vary considerably, which can be misleading to decision-makers.

\section{System dynamic model}

Figure 2 shows the cause-and-effect loops. This model consists of four cause-and-effect loops. Heavy rain- 


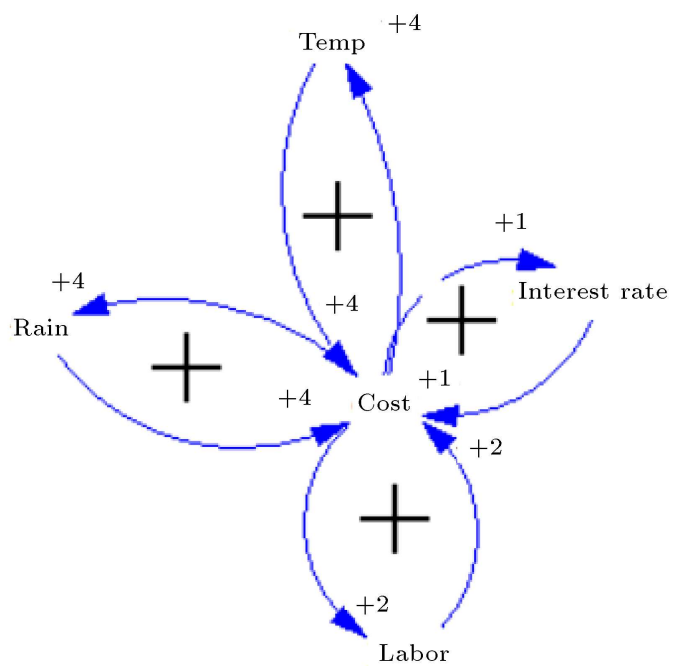

Figure 2. System dynamic model.

fall increases the cost because the project should be stopped and it needs much time to finish the project on time. Increase in costs affects the project time which puts it in high-risk periods and ultimately, it has a positive impact on the case of rainfall. The same holds for the case of temperature. Also, there is a positive relationship between labor and cost. Growth of labor force increases costs.

On the other hand, due to increased costs, greater workforce needs to be present to complete the project as soon as possible. Inflation raises costs and subsequently, rising costs are among the causes of inflation that create a reinforcing loop. All risks affect the costs which, in turn, cause a rise in inflation. As is evident from the numerical results, inflation is identified as a risk with the highest priority.

\section{Conclusion}

Evolutions and developments represent an inevitable part of business environment in which various risks and dangers occur. Therefore, risk management plays a significant role in proper planning for effective decisionmaking under extraordinary circumstances.

In this paper, a new quantitative risk assessment method was presented that considered the cooccurrence of risks. This consideration is important because the co-occurrence of risks intensifies them, or in some cases, reduces their severity. Also, using both metrics "the severity of occurrence" and "the severity of impact" to calculate the risk severity, raises the precision.

To perform risk assessment, the outputs of EMCS and classic MCS were employed as the input of our method. Based on the empirical results achieved by EMCS and MCS considering risks co-occurrence, the inflation was found as the most important risk, orderly followed by cost, rain, temperature, and labor. Without considering their co-occurrence, the risks were prioritized as follows: inflation, rain, temperature, cost, and labor, in order. There was a considerable difference between the results obtained from considering the cooccurrence of risks and neglecting it. According to the experts' opinions, the results achieved by EMCS and MCS considering risks co-occurrence were more logical and applicable than those without considering it. This study also considered the cause-and-effect loops to demonstrate the effect of risks on each other. For future work, it is recommended that fuzzy-based methods be employed for calculating the severity of risks. Also, the co-occurrence of risks in the FMEA method can be considered.

\section{References}

1. Xing, X.-Y., Xiao, Y.-Y. and Huang, Z.-D. "Uncertainty research on economic reliability of equipment based on extended Monte Carlo simulation", Industrial Engineering and Engineering Management (IE\&EM), 2010 IEEE 17Th International Conference on. IEEE (2010).

2. Cagliano, A.C., Grimaldi, S., and Rafele, C. "Choosing project risk management techniques: A theoretical framework", Journal of Risk Research, 18(2), pp. 232248 (2015).

3. Verbano, C. and Venturini, K. "Managing risks in SMEs: A literature review and research agenda", Journal of Technology Management \& Innovation, 8(3), pp. 186-197 (2013).

4. Rezaie, K., Amalnik, M.S., Gereie, A., et al. "Using extended Monte Carlo simulation method for the improvement of risk management: Consideration of relationships between uncertainties", Applied Mathematics and Computation, 190(2), pp. 1492-1501 (2007).

5. Fouladgar, M.M., Yadani-Chamzini, A., and Basiri, M. "Risk evaluation of tunneling projects by fuzzy Topsis", International Conference on Management (2011).

6. Kim, Y.-J. "Monte Carlo vs. fuzzy Monte Carlo simulation for uncertainty and global sensitivity analysis", Sustainability, 9(4), p. 539 (2017).

7. Majd, M., Fatemi, A. and Soltanpanah, H. "The risk analysis of oil projects using fuzzy TOPSIS technique (Case study: 18-inch pipeline repair project from Cheshme Khosh to Ahwaz)", Int. J. Basic Sci. Appl. Res., 3(5), p. 28 (2014).

8. Lyu, H.-M., Sun, W.J., Shen, S.L., et al. "Risk assessment using a new consulting process in fuzzy AHP", Journal of Construction Engineering and Management, $146(3)$, p. 04019112 (2020).

9. Sohrabinejad, A. and Rahimi, M. "Risk determination, 
prioritization, and classifying in construction project case study: Gharb Tehran commercial-administrative complex", Journal of Construction Engineering, 2015, pp. 1-10 (2015).

10. Silvestri, A., De Felice, F., and Petrillo, A. "Multicriteria risk analysis to improve safety in manufacturing systems", International Journal of Production Research, 50(17), pp. 4806-4821 (2012).

11. Huang, Z.D., Chang, W.B., Xiao, Y.Y. and Liu, R. "An extended Monte Carlo method on simulating the development cost uncertainties of aircraft", Advanced Materials Research. Trans Tech Publ., 118, pp. 810814 (2010).

12. Chang, B., Kuo, C., Wu, C.H., et al. "Using fuzzy analytic network process to assess the risks in enterprise resource planning system implementation", Applied Soft Computing, 28, pp. 196-207 (2015).

13. Dehdasht, G., Mohamad Zin, R., Ferwati, M.S., et al. "DEMATEL-ANP risk assessment in oil and gas construction projects", Sustainability, 9(8), p. 1420 (2017).

14. Bamakan, S.M.H. and Dehghanimohammadabadi, M. "A weighted Monte Carlo simulation approach to risk assessment of information security management system", International Journal of Enterprise Information Systems (IJEIS), 11(4), pp. 63-78 (2015).

15. Wang, X., Chan, H.K., Yee, R.W., et al. "A two-stage fuzzy-AHP model for risk assessment of implementing green initiatives in the fashion supply chain", International Journal of Production Economics, 135(2), pp. 595-606 (2012).

16. Zhao, L., Ji, Y., Yao, J., et al. "Quantifying the fate and risk assessment of different antibiotics during wastewater treatment using a Monte Carlo simulation", Journal of Cleaner Production, 168, pp. 626631 (2017).

17. Liu, J., Li, Y.P., Huang, G.H., et al. "Assessment of uncertainty effects on crop planning and irrigation water supply using a Monte Carlo simulation based dualinterval stochastic programming method", Journal of Cleaner Production, 149, pp. 945-967 (2017).

18. Wang, Y.-M. and Elhag, T.M. "A fuzzy group decision making approach for bridge risk assessment", Computers \& Industrial Engineering, 53(1), pp. 137-148 (2007).

19. Naderpour, H., Kheyroddin, A., and Mortazavi, S. "Risk assessment in bridge construction projects in Iran using Monte Carlo simulation technique", Practice Periodical on Structural Design and Construction, 24(4), p. 04019026 (2019).

20. Boateng, P., Chen, Z., and Ogunlana, S.O. "An analytical network process model for risks prioritisation in megaprojects", International Journal of Project Management, 33(8), pp. 1795-1811 (2015).

21. Sharma, S. and Pratap, R. "A case study of risks prioritization using FMEA method", International Journal of Scientific and Research Publications, 3(10), pp. 1-4 (2013).

22. Rezaee, M.J., Yousefi, S., Valipour, M., et al. "Risk analysis of sequential processes in food industry integrating multi-stage fuzzy cognitive map and process failure mode and effects analysis", Computers \& Industrial Engineering, 123, pp. 325-337 (2018).

23. Aqlan, F. and Lam, S.S. "Supply chain risk modelling and mitigation", International Journal of Production Research, 53(18), pp. 5640-5656 (2015).

24. Mhatre, T.N., Thakkar, J., and Maiti, J. "Modelling critical risk factors for Indian construction project using interpretive ranking process (IRP) and system dynamics (SD)", International Journal of Quality \& Reliability Management, 34(9), pp. 1451-1473 (2017).

25. Liu, B. and Sun, F.-H. "Research on the risk assessment method of PPP project based on the improved matter element model", Scientia Iranica, 27(2), pp. 614-624 (2020).

26. Wang, X.-X. and Huang, J.-W. "Risk analysis of construction schedule based on Monte Carlo simulation", Computer Network and Multimedia Technology, CNMT 2009, International Symposium on. IEEE (2009).

27. Rezaie, K., Gereie, A., Ostadi, B., et al. "Safety interval analysis: A risk-based approach to specify low-risk quantities of uncertainties for contractor's bid proposals", Computers \& Industrial Engineering, 56(1), pp. 152-156 (2009).

\section{Biographies}

Bakhtiar Ostadi is an Assistant Professor of Industrial Engineering at Tarbiat Modares University. He received his $\mathrm{PhD}$ in Industrial Engineering from the School of Engineering at Tarbiat Modares University. In addition, he received his MSc from the University of Tehran in Industrial Engineering and his BSc in Applied Mathematics from K.N. Toosi University of Technology. His main areas of research interests include business process reengineering, quality engineering and management, desired organizational capabilities (DOCs) for improvement projects, created organizational capabilities from quality management systems (COC-QMs), marketing, corporate finance, dependability management \& RAMS, and activity-based costing. He has published several articles in national and international academic journals and conferences including International Journals of Production Research, Computers \& Industrial Engineering; Productivity and Quality Management; Business Innovation and Research; Applied Mathematics and Computation; Energy; Biomedical Informatics; Applied Mathematical 
Modelling; Statistical Computation and Simulation; Scientia Iranica; Iranian Journal of Management Studies, etc.

Sara Abbasi Harofteh is an MSc Student at Tarbiat
Modares University. She received BSc in Industrial Engineering from Yazd University. She has several papers in refereed journals and conference proceedings. Her research interests include risk management and risk assessment. 